\title{
论文
}

\section{地磁亚暴期间低高度ENA辐射事件的抵近观测与 反演}

路立 $^{1^{*}}$, Susan MCKENNA-LAWLOR ${ }^{2}$, Jan BALAZ ${ }^{2,3}$

1. 中国科学院国家空间科学中心空间环境探测研究室, 北京 100190 ;

2. Space Technology Ireland, Ltd., Maynooth, Co. Kildare, Ireland;

3. Institute of Experimental Physics, Kosice, Slovakia

*E-mail: luli@nssc.ac.cn

收稿日期：2018-07-18; 收修改稿日期：2018-11-13; 接受日期：2018-11-22; 网络版发表日期：2019-02-15 国家自然科学基金项目(批准号: 41574152)和斯洛伐克科学基金项目(编号: 2-0077-16)

摘要 2004 年11月12日的系列地磁亚暴期间, TC-2卫星上的中性原子成像仪(NUADU)在飞临近地点附近的南、 北极上空观测到低高度能量中性原子(ENA)辐射. 通过对高时空分辨探测数据的反演, 我们首次给出亚暴期间环 电流能量离子沉降和注入过程的全球视场展示, 展示的时间分辨率高达 $1 \mathrm{~min}$. 探测结果表明, 观测到的ENA辐射 主要来自低高度、高纬极区. 由于沉降能量离子沿磁力线运动, 在该区域通过电荷交换产生ENA的几率更大. 因 此, 利用低轨道卫星探测高时间和高空间分辨率的ENA成像数据是监测地磁活动期间环电流离子动力学过程演 化的最佳方法.

关键词低高度ENA辐射, ENA图像反演, 投扶角分布, 离子注入, 离子沉降

\section{1 引言}

Roelof(1987)开创的地磁空间ENA探测数据反演 技术首次建立了ENA图像与赤道环电流离子分布的 直接联系. 这为地磁活动期间磁层环电流的动力学过 程研究提供了有效探测和分析方法. 早期的ENA数据 反演是通过正向模拟实现的. Roelof(1987)利用ISEE-1 卫星上搭载的MEP探测器在1978年9月29日大磁暴期 间收集的粒子数据获取了第一幅ENA图像. 然后, 他通 过反复调整赤道环电流中的离子通量分布模拟出能量 离子通过电荷交换产生的相同ENA分布. 这就证明了
ENA探测图像中包含了赤道环电流区离子通量分布 的信息，从而证明了通过ENA数据反演可以从ENA图 像数据中得到赤道环电流的能量离子分布.

大多数已发表的涉及离子通量分布反演的研究都 是基于搭载在IMAGE卫星上的高能中性原子成像仪 (HENA)的探测数据(例如, Brandt等, 2002a, 2002b; Perez等, 2000, 2001; Zhang等, 2005). 在这些研究中, 为了满足平滑噪声和统计需求, 每幅用于反演的ENA 图像的积分时间都要超过了 $10 \mathrm{~min}$, 在某些情况下甚 至延长到了 $30 \mathrm{~min}$. 考虑到地磁亚暴的时间尺度, 研究 地磁活动中环电流分布和演化需要更高时空分辨的 
ENA图像数据和反演.

提高ENA图像数据的时空分辨率有两种方法，一 种是提高ENA成像探测仪器本身的性能; 另一种方法 是利用抵近ENA辐射源的成像探测数据. 本文使用在 近地点附近采集的ENA图像数据给出高时空分辨率 的研究结果.

\section{2 中性原子成像观测}

由于能量离子倾向于沿磁力线运动, 辐射带结构 与局部磁场形状相同. 能量离子的最大通量在赤道附 近，在高磁纬度的极区略有下降. 这是因为辐射带中 的能量离子与地球上蒸发出来的低能中性原子相互作 用, 通过电荷交换产生ENA, 导致能量离子损失. 换句 话说, 在地磁活动过程中, 观测到的ENA主要来源于低 高度, 高纬度极区(Brandt等, 1997; McComas等, 2012). 以往的探测与仿真结果也都证实了这一点(Roelof,
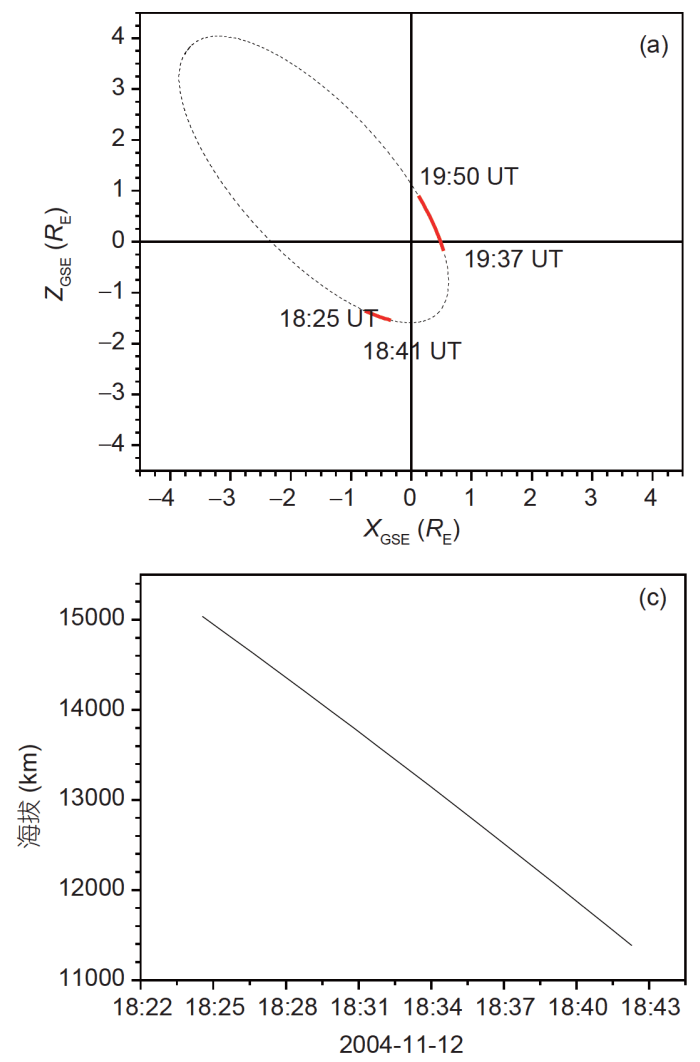

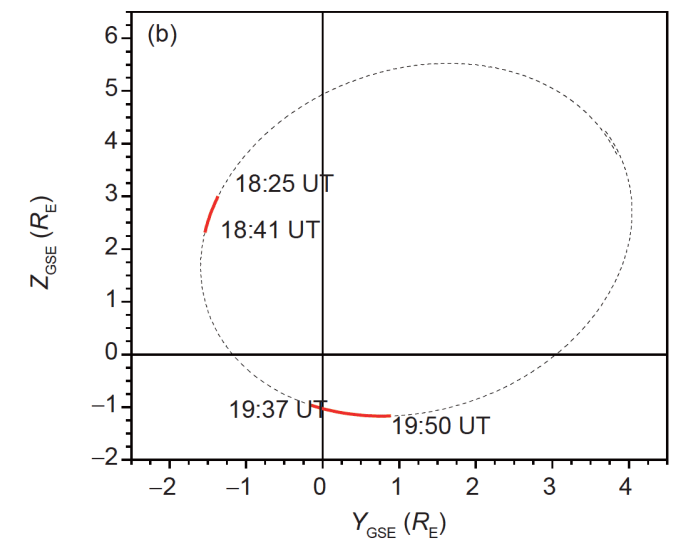

1987; Lu等, 2008b, 2014; Ma等, 2015).

2004年11月 12 日，在一个大磁暴的末期，搭载在 TC-2卫星(Liu等, 2005)上的中性原子成像仪(NUADU) (McKenna-Lawlor等, 2004)在近地点 $(681 \mathrm{~km})$ 附近, 见 图1, 记录了两段ENA辐射增强事件. 两事件之间相隔 约 $1 \mathrm{~h}$ 的NUADU穿越辐射带关机. 图2的原始数据展示 出ENA像素分布几乎布满了 $2 \pi$ 立体角的全部仰角和 方位角. 由于19:37 19:50UT时段的ENA数据在近地点 附近的采集高度为700 3000km(图1d), 图2中(TC-2的 卫星的自旋轴指向黄北极)ENA计数较大的像素仰角 均大于 $90^{\circ}$, 即朝向地球方向, 这表明ENA辐射源在极 区高度(海拔高度的Z分量)小于700km.2006年11月6 日，STEREO/STE掠过夜侧近地点，高度510 840km $(L \leq 1.15)$, 观测到类似的ENA图像(Wang等, 2010).

相应的地磁活动指数由图3给出. 图3a显示IMF南 向, 其中 $0 \leq B_{Z} \leq 8 \mathrm{nT}$ (红色曲线). 图 $3 \mathrm{~d}$ 中的 SYM-H指数, $-60 \sim-80 \mathrm{nT}$, 表征了一个中等偏弱的地磁活动事件. 在

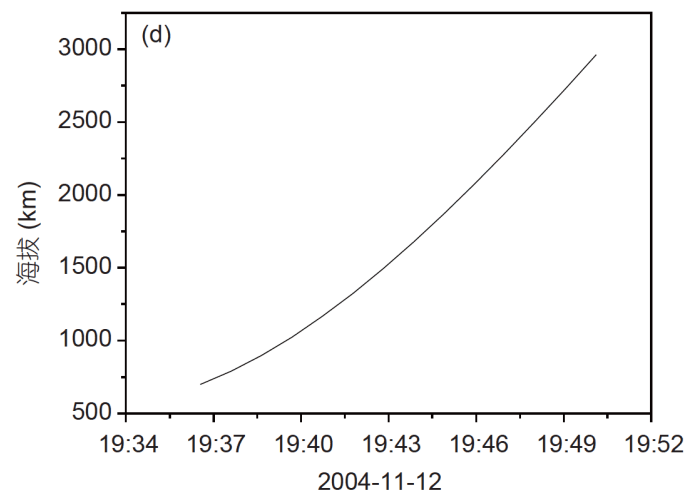

图 122004 年11月12日 1 TC-2卫星在 $X-Z$ 平面(a)和 $Y-Z$ 平面(b)上的轨道、TC-2卫星在18:25 18:41UT(c)和 19:37 19:50UT(d)时 段的海拔高度

(a)和(b)中红线表示采集ENA数据的位置, 对应时间分别标注在图中 


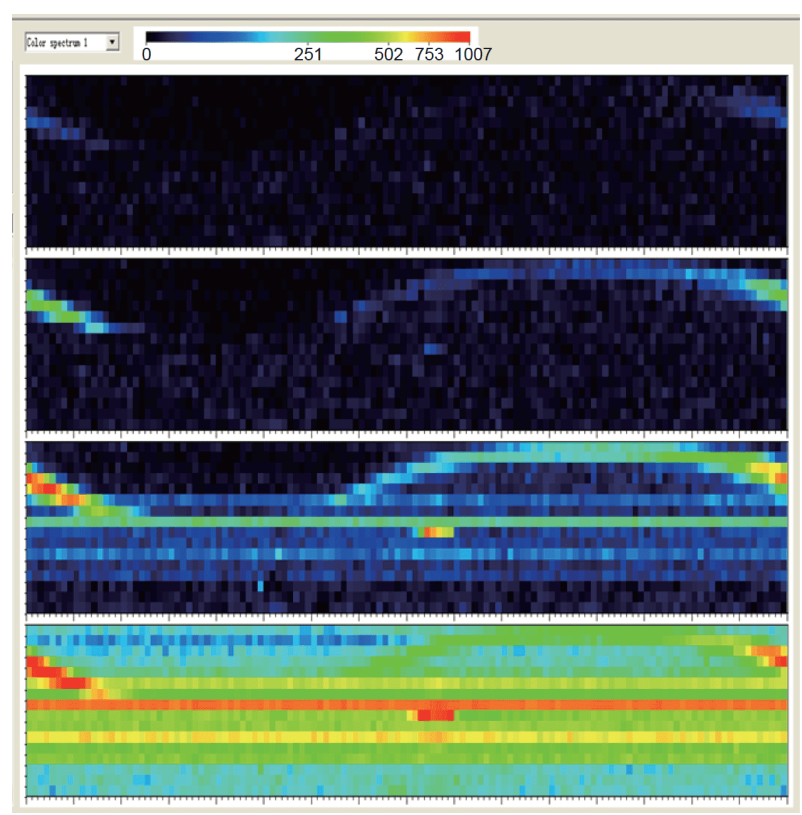

图 2 19:45:24UT, NUADU在4个卫星自旋周里采集的 ENA原始数据图像

横坐标为 $360^{\circ}$ 方位角, 纵坐标为 $180^{\circ}$ 仰角. 从上到下显示了从高到低 的四个能量通道. 顶部的色标显示累积ENA计数

18:25 18:41UT时段, 太阳风动压处于较高的水平(图 $3 \mathrm{~b}$ ), $\mathrm{AE}$ 指数(图3c)增长显示为亚暴的膨胀相. 此后, 当 太阳风动压降低, $\mathrm{AE}$ 指数下降, 表示19:37 19:50UT为 亚暴的恢复相阶段.

NUADU的第二能道 $(50 \sim 81 \mathrm{keV})$ 在亚暴膨胀相和 恢复相期间采集的ENA图像数据，在满足统计需求前 提下分别采用积分时间 $4 \mathrm{~min}$ 和 $64 \mathrm{~s}$ 在角坐标系中展示, 见图4和5. 可以看出, 主要的ENA辐射出现在黄昏到夜 晚时区. 在亚暴膨胀相阶段(图4), ENA辐射增强首先 出现在午夜, 然后向黄昏方向延伸. 在亚暴恢复相阶段 (图5), ENA辐射增强则首先出现在黄昏，然后向午夜 延伸.

图 5 中ENA数据的采集高度仅为图 4 的 $6 \%$ 左右, 见 图 $1 \mathrm{c}$ 和 $1 \mathrm{~d}$. 这使得图 5 中抵近观测数据的时空分辨率远 高于图4. 在图5中, 许多增亮的ENA像素围绕着地球轮 廓, 表现出不同的结构特征. $1 \mathrm{~min}$ 的时间分辨率使我们 能够有机会详细了解在亚暴恢复相的进化过程.

\section{3 环电流离子的投掷角分布}

通常我们考虑投掷角各向异性时，赤道环电流的

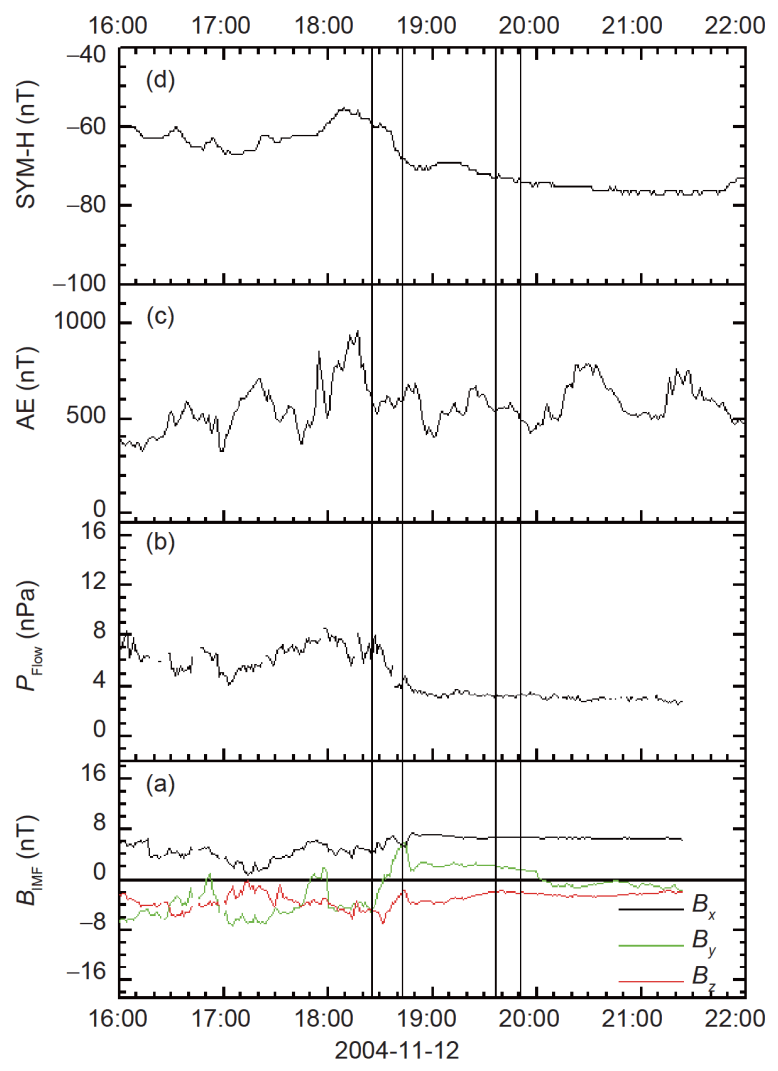

图 32004 年11月 12 日行星际磁场(IMF, (a))、太阳风动压 (b)、AE指数 (c)和SYM-H指数 (d) 的时间变化曲线

ENA数据采集时段(18:25 18:41UT)和(19:37 19:50UT))分别用坚线 分割表示

离子通量可由指数模型表示, 式(1); 其中, 投搠角分布 参照损失雉加以修正(Roelof和Skinner, 2000), 式(2).

$j_{\max 0}^{\mathrm{eq}}(\phi, L, \alpha)=J_{0}^{\mathrm{eq}} \exp \left\{-\left(f_{\phi}+f_{L}+f_{\alpha}\right)\right\}$,

$f_{\alpha}=\left\{\begin{array}{cc}k_{\alpha} \cos ^{2} \alpha_{\mathrm{eq}} & \left(k_{\alpha} \geq 0\right) \\ -k_{\alpha} \cos ^{2} \alpha_{\mathrm{eq}} & \left(k_{\alpha}<0\right)\end{array}\right\}$

$$
+\left\{\begin{array}{cc}
0 & \text { outside-loss-cone } \\
a_{\alpha} & \text { inside-loss-cone }
\end{array}\right\} .
$$

由Kappa模型(Christon等, 1991)推演的环电流离 子通量(Shen和Liu, 2002)可以表示为式(3):

$$
\begin{aligned}
& j_{\text {ion }}(L, \phi, \lambda, E, \alpha)=e j_{0}(L, \phi) \frac{E}{E_{0}}\left(1+\left(\frac{E\left(L / L_{0}\right)^{3}}{\kappa E_{0}}\right)\right. \\
& \left.\times\left[\sin \alpha_{\mathrm{eq}}+\left(1-\sin \alpha_{\mathrm{eq}}\right)\left(L_{0} / L\right)^{0.45}\right]^{2}\right) \cdot
\end{aligned}
$$




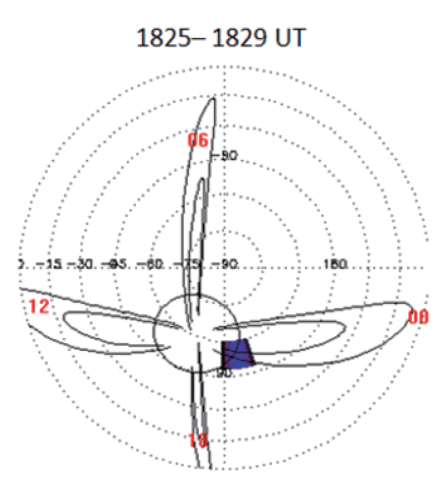

1833-1837 UT

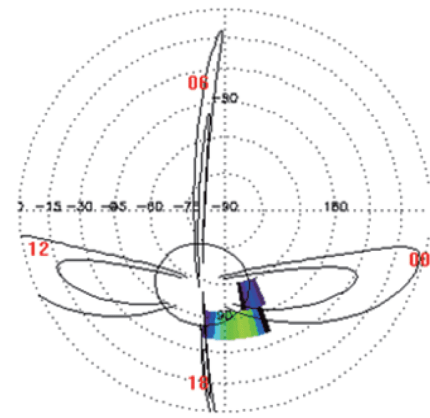

1829-1833 UT
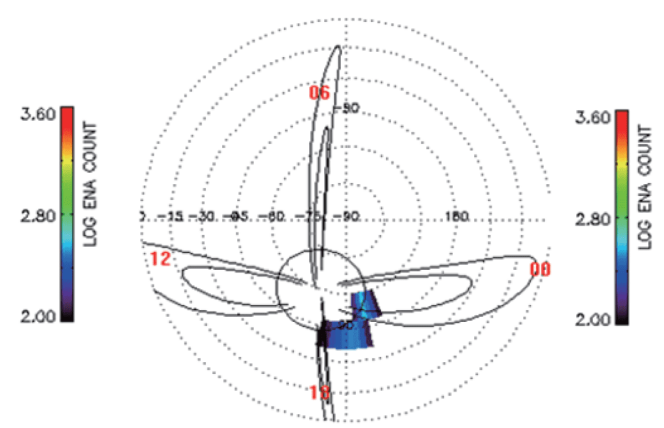

1837-1841 UT

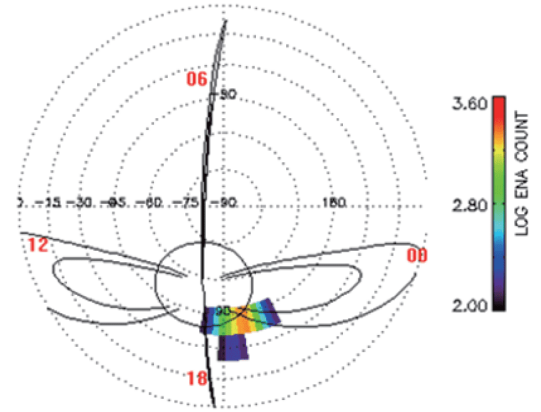

图 42004 年11月 12 日 NUADU/TC-2的50 81 keV 能量通道在18:25 18:41UT期间采集的ENA图像序列

每幅图像的积分时间为 $4 \mathrm{~min}$. 每幅ENA图像中, 仰角用圆形虚线标注, 方位角用虚直线标注. 黑色曲线表示磁壳中 $L=4 、 8$ 的磁力线在ENA探测 图上的投影, 对应的地方时标注为红色. ENA计数的对数色标在每幅图的右侧. ENA图像的采集时间标注在图的上方

图6给出了上述两个模型所对应的归一化离子通 量的投掷角分布. 卫星的局地探测表明环电流能量离 子有时会呈现出较弱的各向异性(Fok等，1996)，所以 在部分经典ENA反演研究模型中采用了各向同性的 假设(Brandt等, 2001, 2002a; Roelof, 1987). 这里我们注 意到上述实用中的投掷角分布假设都是理想化的，忽 略了它在不同时区所表现出不同分布特性的探测真实 情况. 由于在同一个反演模型中无法引入多种投掊角 分布，鉴于Kappa模型的投掷角变化介于其他两种分 布之间，并且适用于赤道日侧环电流的大部分区域, 所以在以下的ENA图像反演中我们采用Kappa模型.

\section{4 亚暴膨胀相的ENA成像数据反演}

由于以上讨论的ENA辐射主要出现在黄昏, 我们 选用Cluster卫星上测量的磁场数据作为参考, Cluster 卫星在黄昏到午夜时区的磁力线拐点内侧飞行(图7b). 在亚暴膨胀相阶段, 18:25 18:41UT, $\left|B_{Z}\right|$ 减小, $B_{X}$ 先增 加一段时间, 然后减小(如图7a所示), 表明该时区的磁
力线被尾向拉伸 $(\mathrm{Xu}$ 等, 2018). 由于第一个绝热不变量 守恒, 式(4)

$\mu=\frac{\mathrm{m} v_{\perp}^{2}}{2 B}=$ const,

$v_{\perp}^{2} \downarrow$, 则 $v_{\|}^{2} \uparrow$. 磁尾能量离子的投掷角通过绝热扩散, 逐渐从“烤饼型” $\left(v_{\perp}^{2}>v_{\|}{ }^{2}\right)$ 演化为“飞蝶型” $\left(v_{\perp}^{2}<v_{\|}{ }^{2}\right)$, 就 像同步轨道探测记录描述的那样(Sauvaud和Winckler, 1980; Fu等, 2011, 2012a; Liu等, 2017a, 2017b). 这时, 环电流能量离子沿磁力线地向运动到低高度高纬极 区, 称之为沉降离子.

利用线性约束反演方法( $\mathrm{Lu}$ 等, 2008a) 获取了亚暴 膨胀相阶段的赤道面环电流离子通量分布(图8). 低高 度ENA辐射与环电流沉降离子相关. 图8显示环电流沉 降离子源于午夜前赤道平面 $L=6$ 附近, 并在 $L$ 方向内、 外延伸. 强离子通量区出现在黄昏到午夜时区, 并有西 向延伸. 图8所示的离子通量大小也反映了沉降离子贡 献的多少. 环电流区域的能量离子通量在磁场偶极化 之前增加的现象(图8)也曾两次在2005年5月15日的大 

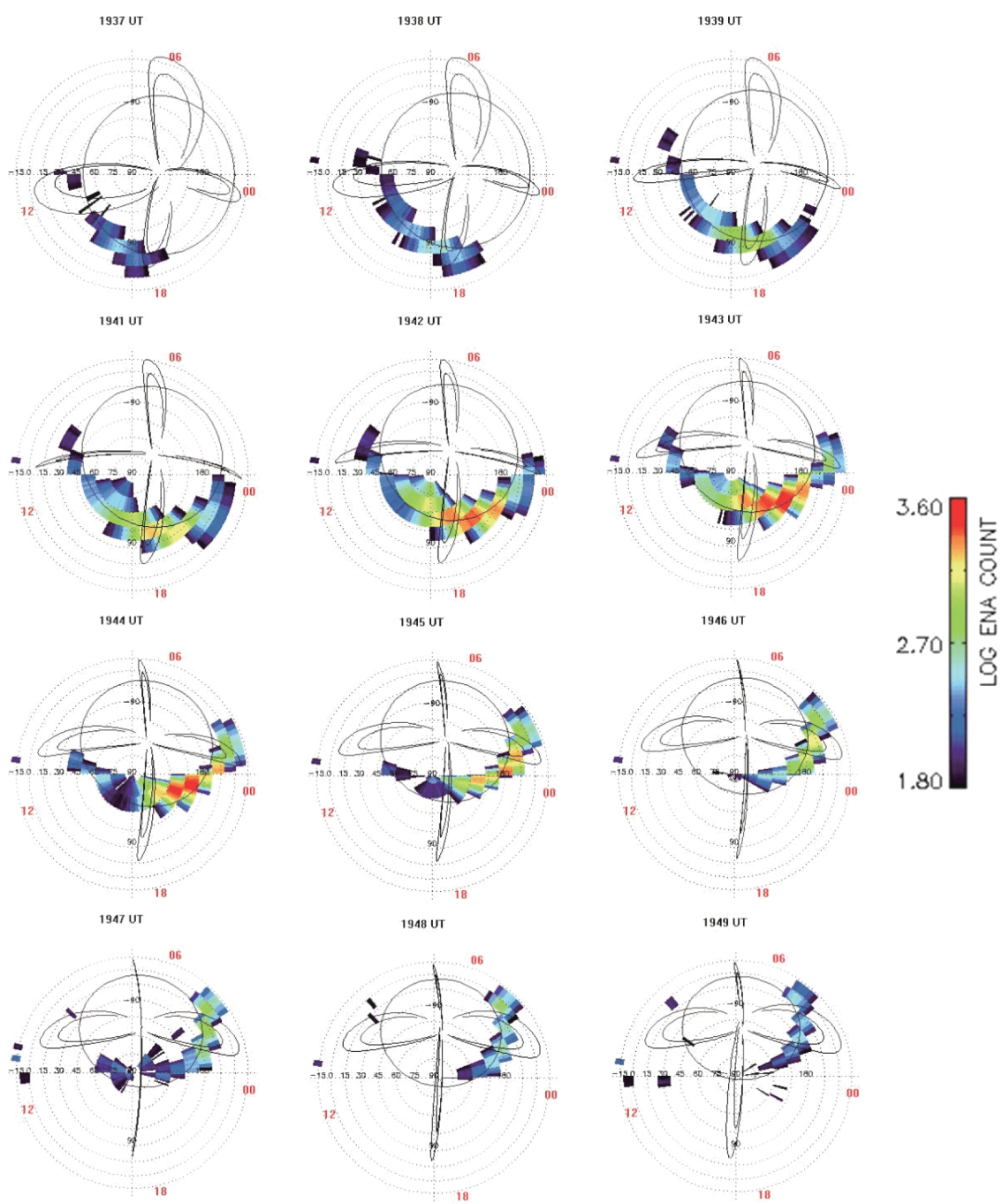

图 52 2004年11月 12日 NUADU/TC-2能量通道50 81 keV在19:37 19:50UT期间采集的ENA图像序列 每幅图像的积分时间为64s. 图中采用的坐标、辅助线和标注与图4相同. 右侧为对数ENA计数色标

磁暴事件中用相同的时间间隔 $(4 \mathrm{~min}$ ) 观测到 $(\mathrm{Lu}$ 等, 2016).

由太阳风从日面压迫地球磁壳 $(\mathrm{Fu}$ 等，2012b, 2012c), 同时晨侧和黄昏侧也受到来自太阳风的压力, 使得 $\mathrm{B} \uparrow$ 伴随有 $T_{\perp}>T_{\|}\left(\right.$图9 $\mathrm{a} \sim \mathrm{c}$ ). 这里 $T_{\perp}$ (或 $\left.T_{\|}\right)$正比于 $v_{\perp}^{2}$ (或 $\left.v_{\|}^{2}\right)$. 由于地球绕太阳公转, 地球上太阳风压的传播 速度: $\overrightarrow{\mathrm{V}}_{\mathrm{SE}}=\overrightarrow{\mathrm{V}}_{\mathrm{S}}-\overrightarrow{\mathrm{V}}_{\mathrm{E}}$. 其中, $\overrightarrow{\mathrm{V}}_{\mathrm{S}}$ 是日地连线方向太阳风压 力的传播速度; $\vec{V}_{\mathrm{E}}$ 是地球公转速度. 这就导致了磁壳 尾向变形的对称中心向黄昏偏移, 从而使环电流沉降 离子, 乃至极光通常会出现在黄昏到午夜时段. 同步
轨道上测量的 $T_{\perp}$ 和 $T_{\|}$的准同步变化, 图 $9 \mathrm{~b}$ 和 $\mathrm{c}$ (时差为 $12 \mathrm{~h})$, 也说明了这一点. 黎明前, 离子通量下降. 但由 于较早的离子注入(图9d), 离子通量仍保持在较高水 平. 这导致能量为 $50 \sim 400 \mathrm{keV}$ 的能量离子密度略有增 加(图9e).

\section{5 亚暴恢复相的ENA 成像数据反演}

在亚暴恢复相阶段(1937 1937UT), Cluster卫星位 于磁场拐点内侧 $\left(B_{Z}<0\right)$. 这里 $B_{X} \downarrow$ 和 $\left|B_{Z}\right| \downarrow$ (图7a), 表明 夜侧磁场的拐点, 乃至整个磁壳, 向地球方向移动. 这 


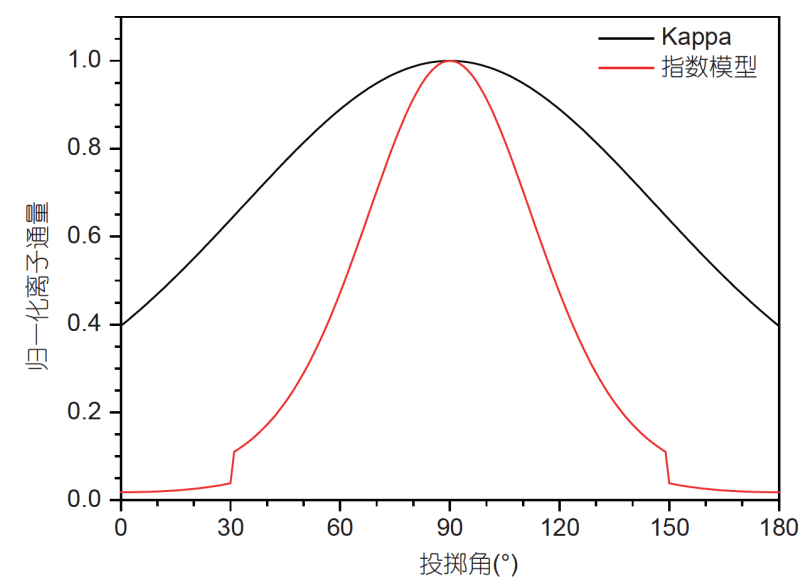

图 6 归一化离子通量的投掷角分布的指数模型和Kappa 模型
提示了磁场偶极化过程. 这时, 夜侧的离子投郑角分布 又恢复了“烤饼型”(如图6所示)。19:37 19:50UT时段, 在地球同步轨道卫星上: 图9b显示了黄昏前高能离子 $(13 \sim 45 \mathrm{keV} / \mathrm{e})$ 的温度, $T_{\|}>T_{\perp}$; 图 $9 \mathrm{c}$ 也显示黎明 前 $T_{\|} \approx T_{\perp}$. 这表明磁场偶极化使得尾向变形的磁壳在 恢复过程中引起晨昏两侧磁壳向外松驰. 那里的磁场 相应减小，离子投掷角向磁场方向扩散。离子通量同 时保持在一个高水平(图9d), 密度下降(图9e), 也提供 了黎明前离子投掷角扩散的间接证据.

利用图5所示的ENA图像数据反演得到的赤道离 子通量分布序列图, 如图10所示. 强离子通量信号(橙 色和红色)最早出现黄昏前, 见图10(19:37 19:38UT). 我们看到这里 $T_{\|}>T_{\perp}$, 预示着与环电流沉降离子有关.
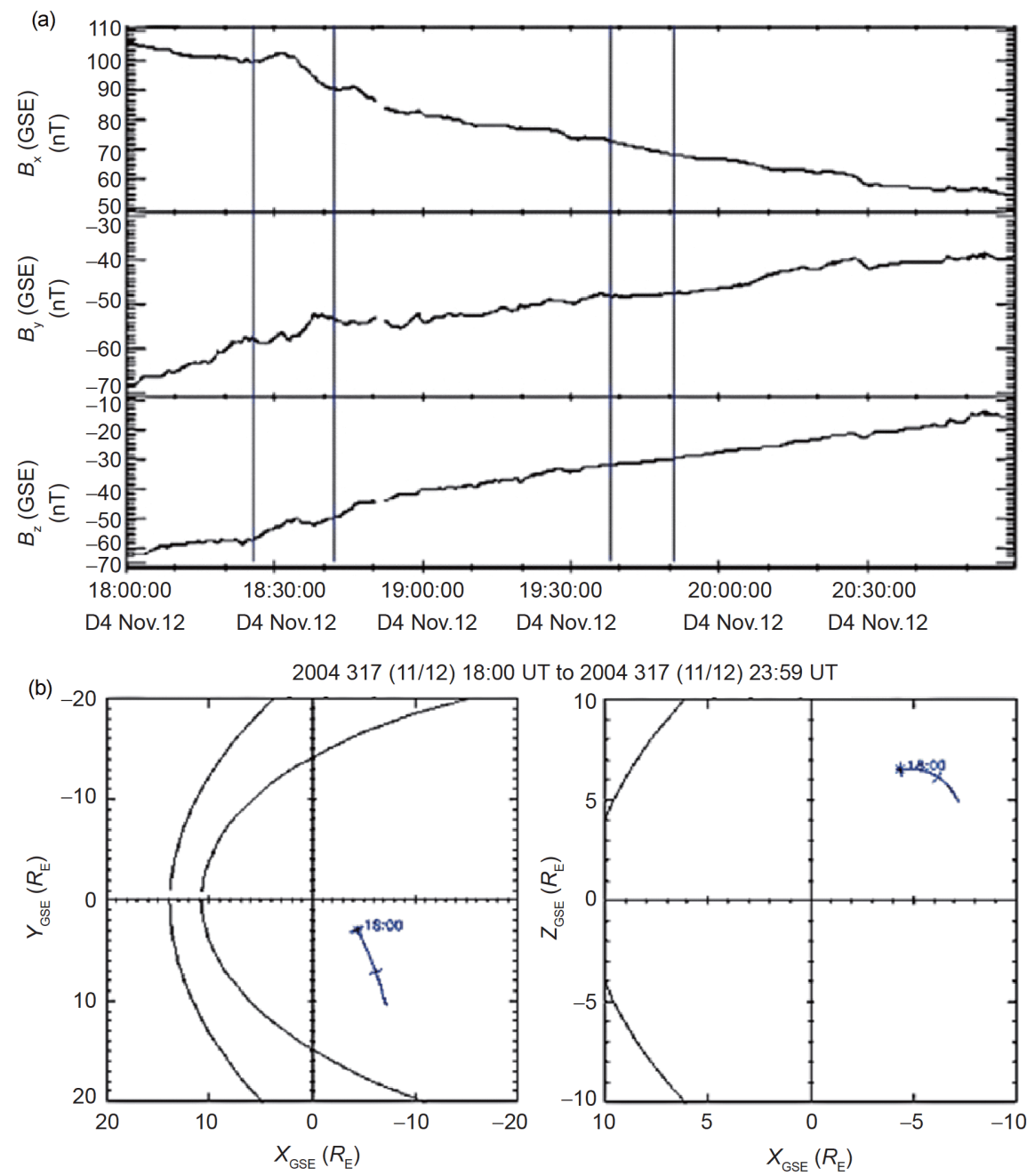

图 7 Cluster卫星测量的磁场(a)和相应的Cluster卫星位置(b) 


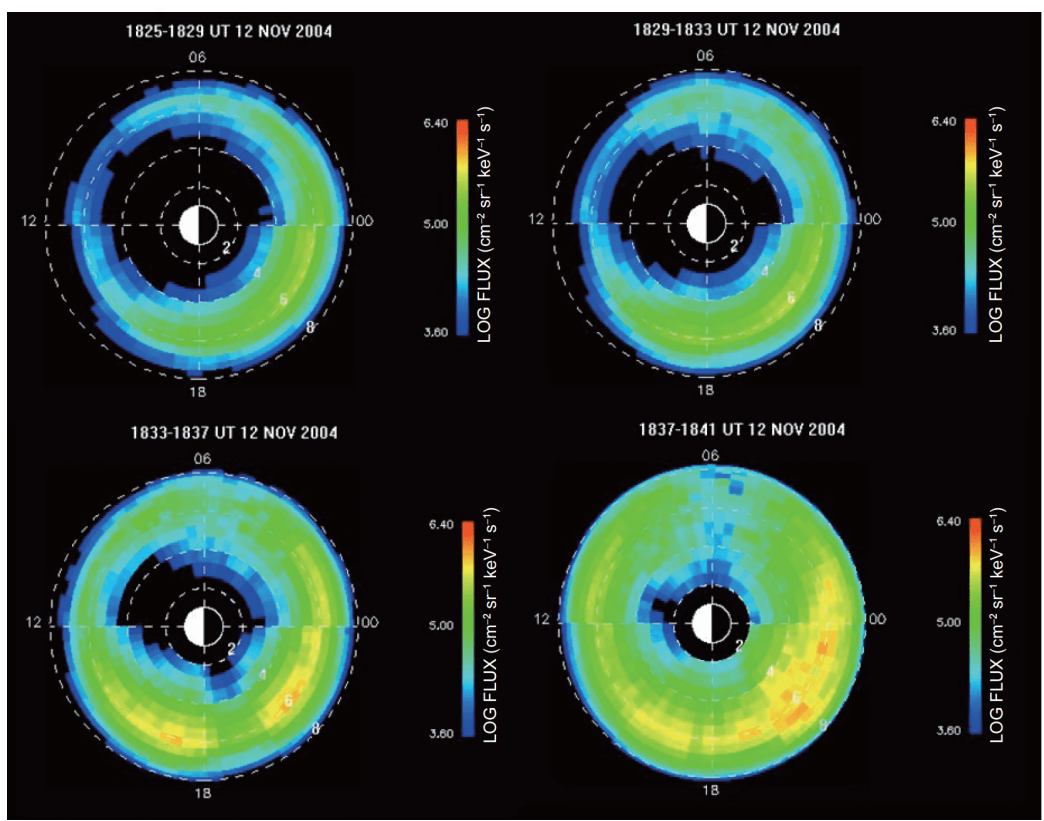

图 8 2004年11月 12日 18:25 18:41UT, 能道50 81 keV, 通过反演获取的赤道离子通量分布序列图 用 $L$-MLT坐标系显示. 每幅图右侧为离子通量对数的色标
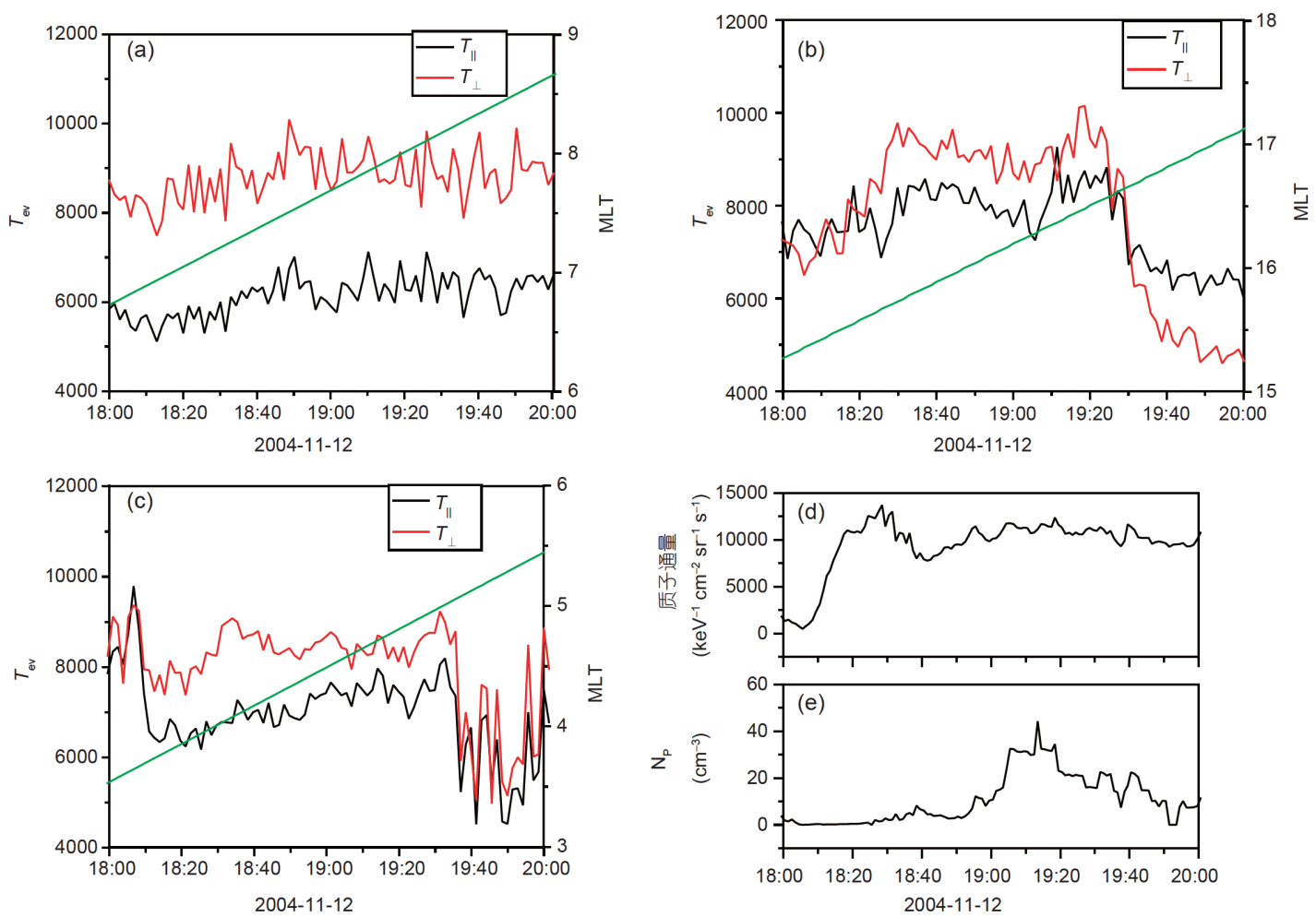

图 9 同步轨道卫星LANL-80(a)、LANL-95(b)和LANL-97(c)上MPA在13 45keV/e能道探测的温度曲线, 及LANL-97卫星 上SPA所得高能 $(50 \sim 400 \mathrm{keV})$ 质子通量 $(\mathrm{d})$ 和质子密度 $(\mathrm{e})$

MPA, 等离子体分析仪; SPA, 同步轨道粒子分析仪. (a) (c)中的绿色线表示磁地方时(MLT) 


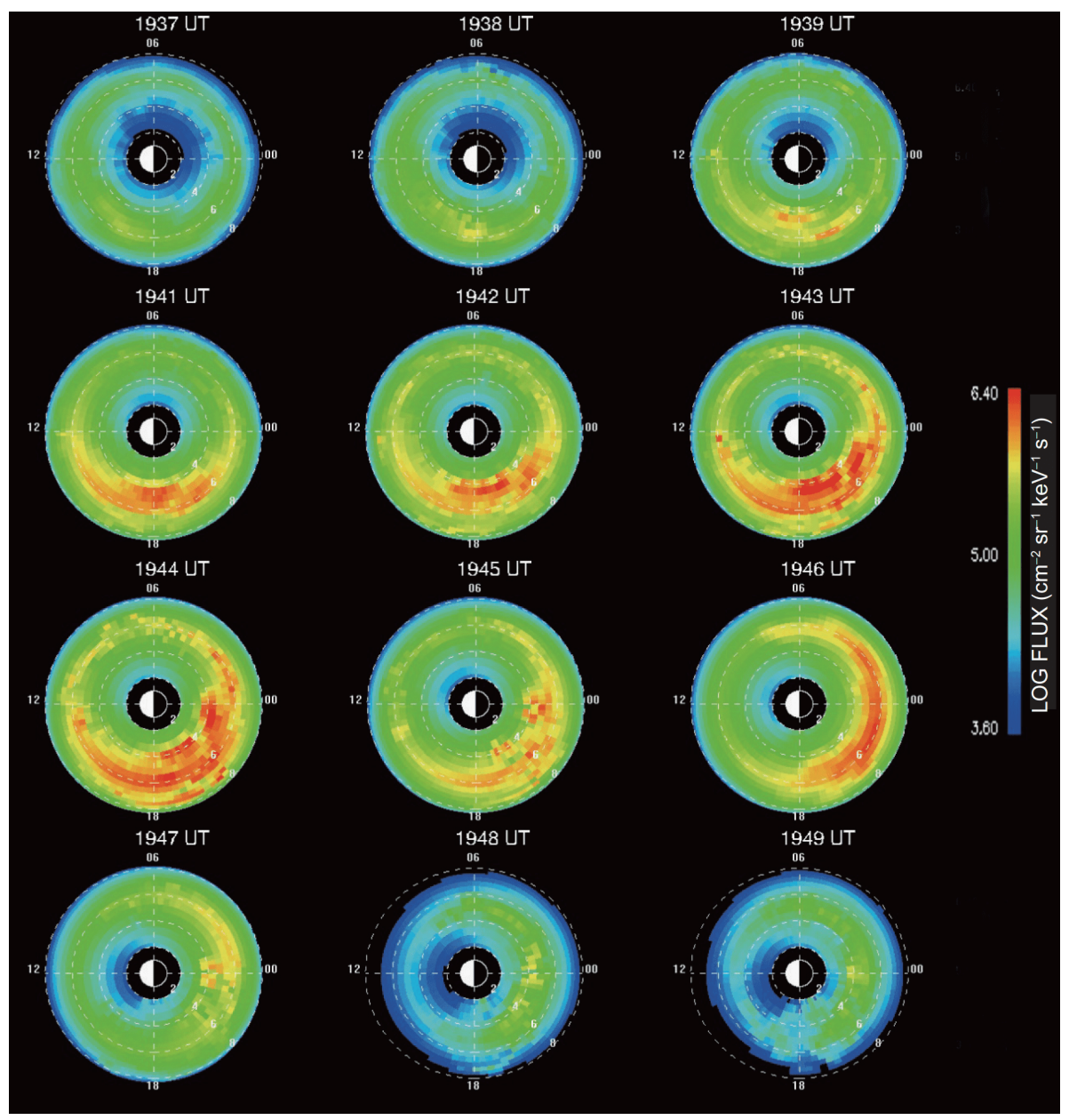

图 102004 年11月 12日 19:37 19:50UT, 能道50 81 keV, 通过反演获取的赤道离子通量分布序列图 用L-MLT坐标系显示. 每幅图顶部为该图数据采集时间. 离子通量对数色标在图形右侧

我们利用图 5 的高空间分辨率数据反演出具有复杂结 构的环电流离子通量分布. 强离子通量双带结构首先 出现在黄昏(19:39UT). 此后, 这种增强的离子通量结 构在黄昏-午夜时区扩展并向东移动(19:41 19:45UT); 最后，该通量分部向地向收缩并在午夜逐渐减弱 (19:46 19:49UT). 图10的赤道离子通量分布序列生动 地描述了环电流离子的注射过程, 就像一个地向的雪 耙还略带东移.

\section{6 讨论和小结}

一般来说，只有投掷角与磁场平行(或反平行)的 赤道环电流离子(称之为沉降离子), 才有机会到达高 纬极区产生低高度ENA辐射. 在特定的投掷角分布前
提下, 赤道环电流离子通量越大所产生的沉降离子也 越多. 所以, 引起低高度ENA辐射增亮有两个原因: 一 个是亚暴膨胀相阶段的环电流能量离子的投掷角扩散 $\left(T_{\|}>T_{\perp}\right)$, 见第4节; 另一个是亚暴恢复相阶段的磁尾能 量离子的注入, 见第5节.

上面描述的两个亚暴事件都是低高度ENA辐射的 例子, 皆产生于来自赤道环电流的沉降离子. 在 19 时 45 分24秒采集的原始ENA数据图像(图2)显示ENA辐射 源的高度小于 $700 \mathrm{~km}$. 对比图 4 和 5 的两组ENA图像, 我 们发现离ENA辐射源越近，采集到的ENA数据的时间 和空间分辨率越高. 由此可以推断，利用一颗低轨道 卫星获取高时空分辨率ENA图像数据是监测地磁活 动期间亚暴环电流演化过程实际可行的最佳方法. 
在亚暴膨胀相阶段, 磁场尾向拉伸, 由于环电流离 子的投郑角扩散而引发离子沉降. 在亚暴恢复相阶段, 磁场偶极化引起夜侧能量离子注入，并伴随有磁壳松 弛引发的黄昏侧能量离子投掷角扩散，所造成的环电 流离子通量分布演化过程首次以 $1 \mathrm{~min}$ 的时间分辨率 予以生动描述.

\section{致谢 对LANL和Cluster卫星团队所提供的探测数据表 示感谢.}

\section{参考文献}

Brandt P C, Barabash S, Norberg O, Lundin R, Roelof E C, Chase C J, Mauk B H, Thomsen M. 1997. ENA imaging from the Swedish micro satellite Astrid during the magnetic storm of 8 February, 1995. Adv Space Res, 20: 1061-1066

Brandt P C, Barabash S, Roelof E C, Chase C J. 2001. Energetic neutral atom imaging at low altitudes from the Swedish microsatellite Astrid: Extraction of the equatorial ion distribution. J Geophys Res, 106: $25731-25744$

Brandt P C, Demajistre R, Roelof E C, Ohtani S, Mitchell D G, Mende S. 2002a. IMAGE/high-energy energetic neutral atom: Global energetic neutral atom imaging of the plasma sheet and ring current during substorms. J Geophys Res, 107: SMP 21-1-SMP 21-13

Brandt P C, Roelof E C, Ohtani S, Mitchell D G, Anderson B. 2002b. IMAGE/HENA: Pressure and current distributions during the 1 October 2002 storm. Adv Space Res, 33: 719-722

Christon S P, Williams D J, Mitchell D G, Huang C Y, Frank L A. 1991. Spectral characteristics of plasma sheet ion and electron populations during disturbed geomagnetic conditions. J Geophys Res, 96: 1-22

Fok M C, Moore T E, Greenspan M E. 1996. Ring current development during storm main phase. J Geophys Res, 101: 15311-15322

Fu H S, Khotyaintsev Y V, André M, Vaivads A. 2011. Fermi and betatron acceleration of suprathermal electrons behind dipolarization fronts. Geophys Res Lett, 38: L16104

Fu H S, Khotyaintsev Y V, Vaivads A, André M, Sergeev V A, Huang S Y, Kronberg E A, Daly P W. 2012a. Pitch angle distribution of suprathermal electrons behind dipolarization fronts: A statistical overview. J Geophys Res, 117: A12221

Fu H S, Cao J B, Mozer F S, Lu H Y, Yang B. 2012b. Chorus intensification in response to interplanetary shock. J Geophys Res, 117: A01203

Fu H S, Cao J B, Zong Q G, Lu H Y, Huang S Y, Wei X H, Ma Y D. 2012c. The role of electrons during chorus intensification: Energy source and energy loss. J Atmos Sol-Terr Phys, 80: 37-47

Liu C M, Fu H S, Xu Y, Cao J B, Liu W L. 2017a. Explaining the rolling-pin distribution of suprathermal electrons behind dipolarization fronts. Geophys Res Lett, 44: 6492-6499

Liu C M, Fu H S, Cao J B, Xu Y, Yu Y Q, Kronberg E A, Daly P W. 2017b. Rapid pitch angle evolution of suprathermal electrons behind dipolarization fronts. Geophys Res Lett, 44: 10116-10124

Liu Z X, Escoubet C P, Pu Z, Laakso H, Shi J K, Shen C, Hapgood M. 2005. The Double Star mission. Ann Geophys, 23: 2707-2712

Lu L, McKenna-Lawlor S, Barabash S, Kudela K, Balaz J, Strharsky I, Liu Z X, Shen C, Cao J B, Brandt P C, Tang C L, Dandouras I. 2008a. Iterative inversion of global magnetospheric ion distributions using energetic neutral atom (ENA) images recorded by the NUADU/TC2 instrument. Ann Geophys, 26: 1641-1652

Lu L, Mckenna-Lawlor S, Barabash S, Balaz J, Liu Z X, Shen C, Cao J B, Tang C L. 2008b. Iterative inversion of global magnetospheric information from energy neutral atom (ENA) images recorded by the TC-2/NUADU instrument. Sci China Ser E-Technol Sci, 51: 1731-1744

Lu L, McKenna-Lawlor S, Balaz J, Shi J, Yang C, Luo J. 2014. Technical configuration and simulation of the NAIS-H for the MIT mission (in Chinese). Chin J Space Sci, 34: 341-351

Lu L, McKenna-Lawlor S, Cao J B, Kudela K, Balaz J. 2016. The causal sequence investigation of the ring current ion-flux increasing and the magnetotail ion injection during a major storm. Sci China Earth Sci, 59: 129-144

Ma S Y, Yan W N, Xu L. 2015. Tomographic reconstruction of storm time RC ion distribution from ENA images on board multiple spacecraft. J Geophys Res-Space Phys, 120: 9334-9354

McKenna-Lawlor S, Balaz J, Strharsky I, Barabash S, Brinkfeldt K, Li L, Shen C, Shi J, Zong Q, Kudela K, Fu S, Roelof E C, son Brandt P C, Dandouras I. 2004. The energetic NeUtral atom detector unit (NUADU) for China's Double Star Mission and its calibration. Nucl Inst Method Phys Res Sect A, 530: 311-322

McComas D J, Buzulukova N, Connors M G, Dayeh M A, Goldstein J, Funsten H O, Fuselier S, Schwadron N A, Valek P. 2012. Two wideangle imaging neutral-atom spectrometers and interstellar boundary explorer energetic neutral atom imaging of the 5 April 2010 substorm. J Geophys Res, 117: A03225

Perez J D, Fok M C, Moore T E. 2000. Deconvolution of energetic neutral atom images of the earth's magnetosphere. Space Sci Rev, 91: $421-436$

Perez J D, Kozlowski G, C:son-Brandt P, Mitchell D G, Jahn J M, Pollock C J, Zhang X X. 2001. Initial ion equatorial pitch angle distributions from medium and high energy neutral atom images obtained by IMAGE. Geophys Res Lett, 28: 1155-1158 
Roelof E C. 1987. Energetic neutral atom image of a storm-time ring current. Geophys Res Lett, 14: 652-655

Roelof E C, Skinner A J. 2000. Extraction of ion distributions from magnetospheric ENA and EUV images. Space Sci Rev, 91: 437-459

Sauvaud J A, Winckler J R. 1980. Dynamics of plasma, energetic particles, and fields near synchronous orbit in the nighttime sector during magnetospheric substorms. J Geophys Res, 85: 2043-2056

Shen C, Liu Z X. 2002. Properties of the neutral energetic atoms emitted from Earth's ring current region. Phys Plasmas, 9: 39843994
Wang L, Lin R P, Parks G K, Brandt P C, Roelof E C, Sample J G, Eastwood J P, Larson D E, Curtis D W, Luhmann J G. 2010. Energetic, 5-90 keV neutral atom imaging of a weak substorm with STEREO/STE. Geophys Res Lett, 37: L08107

Xu Y, Fu H S, Norgren C, Hwang K J, Liu C M. 2018. Formation of dipolarization fronts after current sheet thinning. Phys Plasmas, 25 : 072123

Zhang X X, Perez J D, Chen T, Wang C, Brandt P C, Mitchell D G, Wang Y L. 2005. Proton temperatures in the ring current from ENA images and in situ measurements. Geophys Res Lett, 32: L16101

(责任编委: 傅绥燕) 